\title{
Identification of cucurbit chlorotic yellows virus P4.9 as a possible movement protein
}

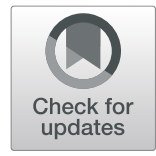

Ying Wei, Yajuan Shi, Xaioyu Han, Siyu Chen, Honglian Li, Linlin Chen, Bingjian Sun and Yan Shi

\begin{abstract}
Background: Cucurbit chlorotic yellows virus (CCYV) is a bipartite cucurbit-infecting crinivirus within the family Closteroviridae. The crinivirus genome varies among genera. P4.9 is the first protein encoded by CCYV RNA2. P5, which is encoded by LIYV, is necessary for efficient viral infectivity in plants; however, it remains unknown whether CCYV P4.9 is involved in movement.

Finding: In this study, we used green fluorescent protein (GFP) to examine the intracellular distribution of P4.9-GFP in plant cells, and observed fluorescence in the cytoplasm and nucleus. Transient expression of P4.9 was localized to the plasmodesmata. Co-infiltration of agrobacterium carrying binary plasmids of P4.9 and GFP facilitated GFP diffusion between cells. Besides P4.9 was able to spread by itself to neighboring cells, and co-localized with a marker specific to the endoplasmic reticulum, HDEL-mCherry, but not with the Golgi marker Man49-mCherry.
\end{abstract}

Conclusions: Together, these results demonstrate that CCYV P4.9 is involved in cell-cell movement.

Keywords: CCYV, P4.9, Movement

\section{Main text}

Plant viruses are small obligate intracellular parasites that live in the symplast of their hosts. Plant virus movement is an essential component of complete viral infection. Cell-cell movement, through the cell walls separating adjacent cells, involves movement proteins (MPs) that alter the size exclusion limit (SEL) of the plasmodesmata $(\mathrm{PD})$, enabling transport of the viral genome into adjacent cells $[1,2]$.

Cucurbit chlorotic yellows virus (CCYV) is a cucurbitinfecting crinivirus within the family Closteroviridae [35]. Members of this family possess the largest, most complex single-stranded RNA (ssRNA) genomes, which vary in size from ca. 15 to $20 \mathrm{~kb}$ [6]. Like most members of the Crinivirus genus, CCYV has a bipartite genome. The crinivirus genome varies among species in the RNA1 3' region downstream of the RNA-dependent RNA polymerase, where the number of open reading frames (ORFs) varies from zero to three. CCYV RNA2 contains eight ORFs, which encode P4.9, HSP70h, P6.5, P59, P9, CP (major coat protein), CPm (minor coat protein), and P26. P4.9, HSP70h, P59, CP, and CPm are

\footnotetext{
* Correspondence: shiyan00925@126.com

College of Plant Protection, Henan Agricultural University, Zhengzhou 450002, China
}

quintuple gene blocks that are conserved among all members of the family Closteroviridae [7].

Beet yellows virus (BYV) is a well-studied closterovirus that requires five proteins for cell-cell movement: P6, HSP70h, P64, CP, and CPm [8-10]. Similarly, Citrus tristeza virus (CTV) proteins involved in viral assembly also have functions in viral spread; these include HSP70h, $\mathrm{P} 61, \mathrm{CP}$, and minor $\mathrm{CP}(\mathrm{CPm})$. Two other proteins, P6 and $\mathrm{P} 33$, are not required for assembly but are involved in virus movement $[11,12]$. A non-virion protein, P26, which is encoded by lettuce infectious yellows crinivirus, is PD-localized and essential for viral systemic infection [13]. Small proteins P5 and P9, which are encoded by LIYV RNA2, are indispensable for efficient viral infectivity in plants [14]. Movement proteins differ among cloteroviruses; therefore, the objective of this study was to determine whether the CCYV protein P4.9 is a movement protein.

Based on an amino acid sequence analysis performed using the PSORT online tool, we hypothesized that the localization of P4.9 is extracellular, including the cell wall. To determine the intracellular localization of P4.9, we cloned the protein by fusion to the $\mathrm{C}$-terminus of green fluorescent protein (GFP). To construct P4.9-GFP, we amplified the polymerase chain reaction (PCR) 
product using the primer pair BPp4.9F/BPp4.9R and BDp4.9 as template (Additional file 1: Table S1), gateway technology, and the pEarlyGate 103 vector [15]. The resulting clone was transformed into Agrobacterium tumefaciens strain GV3101 for agroinfiltration. P4.9-GFP was visualized using laser scanning confocal microscopy (LSCM) at 2 days post-inoculation (dpi). GFP fluorescence was observed in the cytoplasm and nucleus (Fig. 1a).

To further characterize the intracellular localization pattern, we treated Nicotiana benthamiana leaves expressing P4.9-GFP with a cell wall degrading enzyme to obtain protoplast, as previously described [11]; localization within the protoplast was consistent with our microscopy results (Fig. 1b). The fluorescing tubules protruding from the cell surface was not found as decribed for Citrus tristeza virus (CTV) p33 by Bak (2015). To determine the PD localization of P4.9, we used the callose-binding plasmodesmata marker dye aniline blue. We agrofiltrated $0.1 \mathrm{mg} / \mathrm{mL}$ aniline blue into leaves for $2 \mathrm{~h}$, and then detached the leaves for fluorescence observation. Fluorescence signals were individually visualized using LSCM; we found that P4.9 accumulated in punctate spots along the cell periphery (Fig. 1c), which is characteristic of PD localization. We then demonstrated co-localization of these sites using aniline blue, confirming that P4.9 is PD-localized (Fig. 1c).

A distinguishing property of MPs is the ability to increase the SEL and enhance cell-to-cell trafficking. To construct a P4.9 binary expression vector, we amplified its PCR product using primer pair pGDP4.9PstIF/ pGDP4.9BamHIR (Additional file 1: Table S1), digested

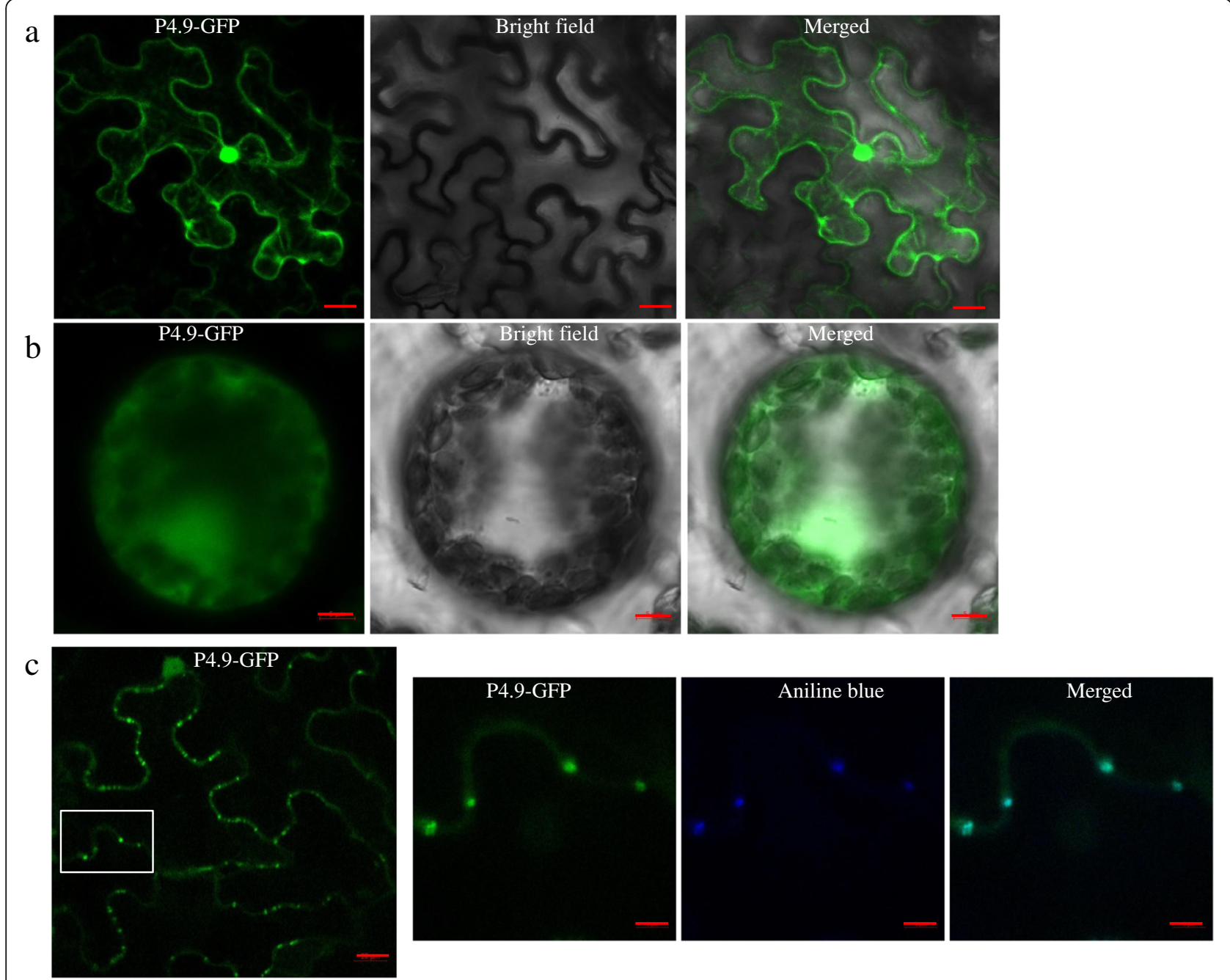

Fig. 1 (a) Subcellular localization of P4.9-GFP, constructed using green fluorescent protein (GFP), was observed in Nicotiana benthamiana leaves at 2 days post-inoculation (dpi). Bar, $20 \mu \mathrm{m}$. b Distribution of P4.9-GFP in protoplasts isolated from agrobacterium-infiltrated N. benthamiana leaves at 2 dpi. Bar, $5 \mu \mathrm{m}$. c P4.9-GFP accumulated in punctate spots along the cell periphery, co-localized with the plasmodesmata marker dye aniline blue at 2 dpi. Bars, $20 \mu \mathrm{m}$ (left) and $5 \mu \mathrm{m}$ (right) 
it using PstI and BamHI, and cloned it into the pGDMyc vector to generate pGDP4.9. Agrobacteria carrying the GFP binary plasmid was diluted 1000-fold, mixed with agrobacterium cultures carrying binary plasmid of P4.9 or GUS, and co-infiltrated into $N$. benthamiana leaves. Leaf samples were viewed for GFP fluorescence 2 days post-infiltration using a fluorescence microscope (ECLIPSE Ti-S, Nikon) at $10 \times$ magnification. GFP fluorescence was mainly restricted to single cells when co-expressed with GUS. P4.9 promoted the diffusion of GFP fluorescence (Fig. 2a). We then estimated the number of cells expressing GFP. Compared with GUS control, P4.9 co-expression with GFP promoted GFP movement. Significantly more cell clusters expressed GFP in 2-3 cells and > 4 cells than in the control (Table 1). To further measure the intercellular capacity of P4.9, agrobacterium carrying P4.9-GFP or YFP control was diluted 4000-fold. YFP was retained in individual epidermal cells, whereas P4.9-GFP spread to neighboring cells (Fig. 2b).
Table 1 P4.9 promoted cell-cell movement of free GFP in Nicotiana benthamiana. Asterisks denote significant differences compared with the results of GUS $(P<0.05)$

\begin{tabular}{lllll}
\hline & 1 cells & $2-3$ cells & $4-5$ cells & Total foci counted \\
\hline P4.9 & $148(56.7 \%)^{*}$ & $103(39.5 \%)^{*}$ & $10(3.8 \%)^{*}$ & 261 \\
GUS & $93(78.2 \%)$ & $25(21 \%)$ & $1(0.8 \%)$ & 119 \\
\hline
\end{tabular}

The endoplasmic reticulum (ER) membrane has been reported to play essential roles in MP and virus cellcell movement by diffusing between cells through the PD $[16,17]$. Therefore, P4.9-GFP was co-expressed with the ER marker HDEL-mCherry in $N$. benthamiana leaves; P4.9 was co-localized with the ER marker (Fig. 2c). We also examined the localization of P4.9 in the Golgi apparatus using the marker Man49-mCherry (Arabidopsis Biological Resource Center, ABRC). LSCM showed that P4.9-GFP did not co-localize with the Golgi apparatus (Fig. 2d), indicating that intracellular

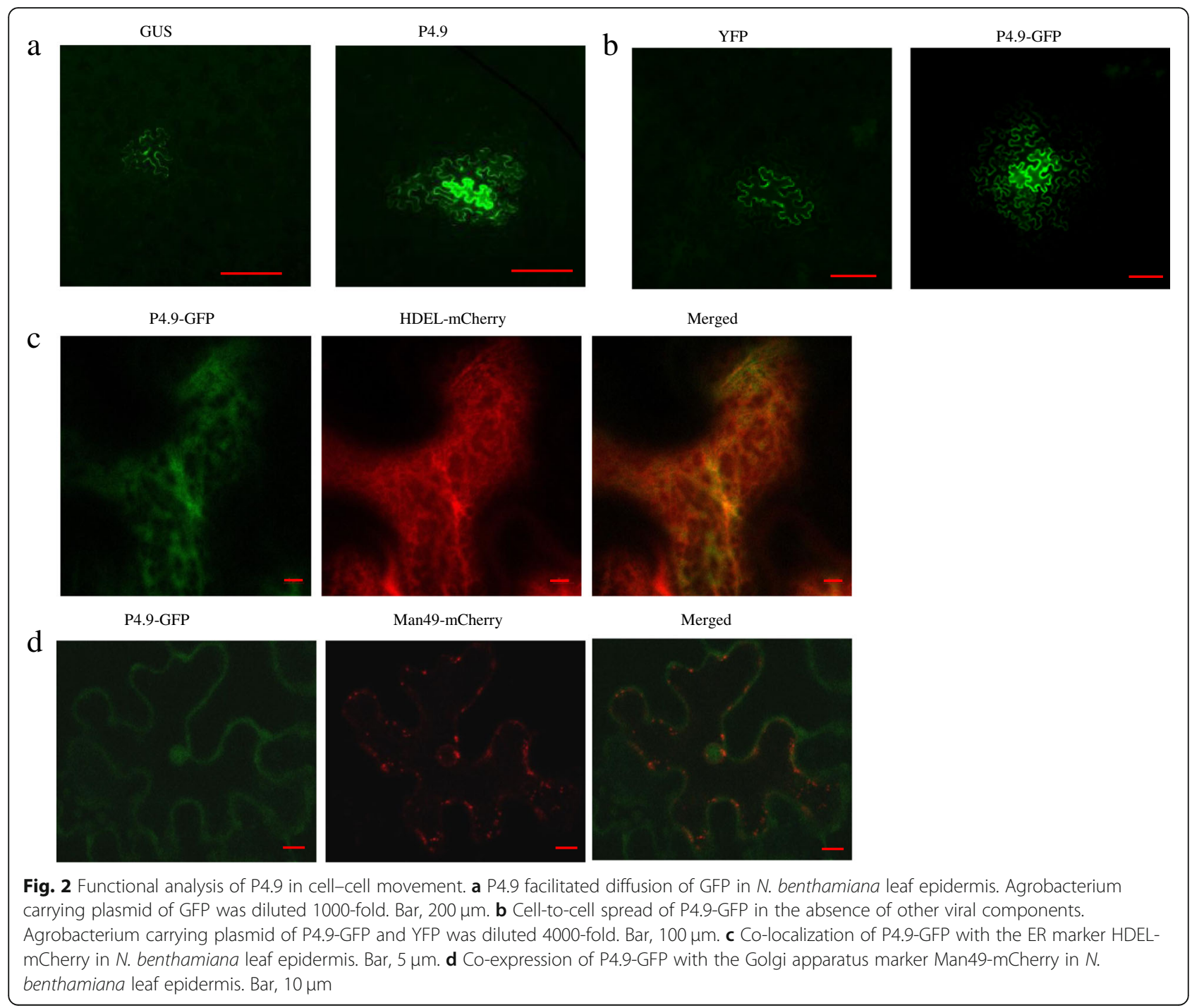


trafficking of P4.9 is independent of the ER-Golgi secretory pathway.

Different plant viruses use different host cell transport machinery to move from one cell to another through the PD [17-21]. A previous study of LIYV P5 and P9 showed that neither protein was essential for viral infection, but that knocking out either protein led to debilitated LIYV infection [14], suggesting that these two proteins may participate in viral movement. Similarly, the P6 protein of closteroviruses BYV and CTV has been found to be involved in viral movement [10-12].

In this study, we found that transient expression of P4.9 was localized to the PD, and facilitated GFP diffusion between cells. P4.9 was co-localized with the ERspecific marker ER-Cherry but not the Golgi marker Manl-Cherry. Our results provide direct evidence that the CCYV protein P4.9 is involved in cell-cell movement. To our knowledge, this is the first such demonstration for a member of the genus Crinivirus.

\section{Additional file}

Additional file 1: Table S1. Primers used in this study. Restriction sites were underlined, and start and stop codons were shown in italics. The English in this document has been checked by at least two professional editors, both native speakers of English. For a certificate, please see http://www.textcheck.com/certificate/JDS64O. (PDF 400 kb)

\section{Abbreviations}

CCY: Cucurbit chlorotic yellows virus; GFP: Green fluorescent protein MP: Movement proteins; PD: Plasmodesmata; SEL: Size exclusion limit

\section{Acknowledgements}

The authors wish to thank Dr. Min Zhu from Nanjing agricultural university for providing the plasmid Man49-mCherry and Dr. Hangjun Sun from Henan agricultural university for providing the plasmid HDEL-mCherry. We thank professor Zaifeng Fan for kindly providing pGDMyc.

\section{Authors' contributions}

YS and YW designed the experiment. YW, YJS and XH performed the experiment and data analysis. YS wrote paper. LC, HL, BS, SC were involved in discussion. All authors read and approved the final manuscript.

\section{Funding}

Financial support was provided by National Natural Science Foundation of China (31701944).

\section{Availability of data and materials}

Not applicable.

Ethics approval and consent to participate

Not applicable.

\section{Consent for publication}

All the authors consent to publish.

Competing interests

The authors declare that they have no competing interests.
Received: 31 March 2019 Accepted: 14 June 2019

Published online: 20 June 2019

\section{References}

1. Wolf S, Deom CM, Beachy RN, Lucas WJ. Movement protein of tobacco mosaic virus modifies plasmodesmatal size exclusion limit. Science. 1989; 246:377-9.

2. Mann KS, Bejerman N, Johnson KN, Dietzgen RG. Cytorhabdovirus P3 genes encode 30K-like cell-to-cell movement proteins. Virology. 2016;489:20-33.

3. Gu QS, Liu YH, Wang YH, Huangfu WG, Gu HF, et al. First report of cucurbit chlorotic yellows virus in cucumber, melon, and watermelon in China. Plant Dis. 2011;95:1168.

4. Huang LH, Tseng HH, Li JT, Chen TC. First report of cucurbit chlorotic yellows virus infecting cucurbits in Taiwan. Plant Dis. 2010;94:1168.

5. Okuda M, Okazaki S, Yamasaki S, Okuda S, Sugiyama M. Host range and complete genome sequence of cucurbit chlorotic yellows virus, a new member of the genus Crinivirus. Phytopathology. 2010;100:560-6.

6. Martelli IM, Agranovsky AA, Bar-Joseph M, Boscia D, Candresse T, et al. Closteroviridae. In: Andrew MQ, King MJA, Carstens EB, Lefkowitz EJ, editors. Virus taxonomy classification and nomenclature of viruses ninth report of the international committee taxonomy of viruses. USA: Elsevier Inc:; 2012. p. 987-1001.

7. Dolja W, Kreuze JF, Valkonen JP. Comparative and functional genomics of closteroviruses. Virus Res. 2006;117:38-51.

8. Alzhanova DV, Hagiwara Y, Peremyslov W, Dolja W. Genetic analysis of the cell-to-cell movement of beet yellows closterovirus. Virology. 2000; 268:192-200

9. Peremyslov W, Hagiwara Y, Dolja W. HSP70 homolog functions in cell-tocell movement of a plant virus. Proc Natl Acad Sci U S A. 1999;96:14771-6.

10. Peremyslov W, Pan YW, Dolja W. Movement protein of a closterovirus is a type III integral transmembrane protein localized to the endoplasmic reticulum. J Virol. 2004;78:3704-9.

11. Bak A, Folimonova SY. The conundrum of a unique protein encoded by citrus tristeza virus that is dispensable for infection of most hosts yet shows characteristics of a viral movement protein. Virology. 2015;485:86-95.

12. Tatineni S, Robertson CJ, Garnsey SM, Bar-Joseph M, Gowda S, et al. Three genes of citrus tristeza virus are dispensable for infection and movement throughout some varieties of citrus trees. Virology. 2008:376:297-307.

13. Qiao W, Medina V, Kuo YW, Falk BW. A distinct, non-Virion plant virus movement protein encoded by a Crinivirus essential for systemic infection. MBio. 2018a;9(6):e02230-18.

14. Qiao W, Helpio EL, Falk BW. Two Crinivirus-conserved small proteins, P5 and $\mathrm{P9}$, are indispensable for efficient lettuce infectious yellows virus infectivity in plants. Viruses. 2018b;10. https://doi.org/10.3390/v10090459.

15. Earley KW, Haag JR, Pontes O, Opper K, Juehne T, et al. Gatewaycompatible vectors for plant functional genomics and proteomics. Plant J. 2006:45:616-29.

16. Guenoune-Gelbart D, Elbaum M, Sagi G, Levy A, Epel BL. Tobacco mosaic virus(TMV) replicase and movement protein function synergistically in facilitating TMV spread by lateral diffusion in the plasmodesmal desmotubule of Nicotiana benthamiana. Mol Plant-Microbe Interact. 2008; 21:335-45.

17. Feng Z, Xue F, Xu M, Chen X, Zhao W, et al. The ER-membrane transport system is critical for intercellular trafficking of the NSm movement protein and tomato spotted wilt tospovirus. PLoS Pathog. 2016;12:e1005443.

18. Harries PA, Schoelz JE, Nelson RS. Intracellular transport of viruses and their components: utilizing the cytoskeleton and membrane highways. Mol Plant-Microbe Interact. 2010;23:1381-93.

19. Niehl A, Heinlein M. Cellular pathways for viral transport through plasmodesmata. Protoplasma. 2011;248:75-99.

20. Ueki S, Citovsky $V$. To gate, or not to gate: regulatory mechanisms for intercellular protein transport and virus movement in plants. Mol Plant. 2011:4:782-93.

21. Wang A. Dissecting the molecular network of virus-plant interactions: the complex roles of host factors. Annu Rev Phytopathol. 2015;53:45-66.

\section{Publisher's Note}

Springer Nature remains neutral with regard to jurisdictional claims in published maps and institutional affiliations. 